\title{
La observación desde la teoría coherentista de la justificación
}

\section{Observation from the Perspective of the Coherence Theory of Justification}

\author{
José Rubén Orantes García \\ https://orcid.org/0000-0001-7409-3500 \\ Universidad Nacional Autónoma de México \\ Centro de Investigaciones Multidisciplinarias sobre Chiapas y la Frontera Sur
}

Bonjour, Laurence. (2019). Epistemología: problemas clásicos y respuestas contemporáneas, (traducido por Manuel Arnulfo Cañas Muñoz). Tuxtla Gutiérrez, Chiapas: Universidad Pedagógica Nacional, Unidad 071, 396 pp., ISBN: 978-6078471768.

Resumen:

El texto refleja la convicción de Laurence Bonjour de que el lugar para comenzar cualquier discusión sobre las teorías del conocimiento es a partir de los problemas clásicos, comenzando y centrando su interés en Descartes. Solo después de registrar las bases para comprender la epistemología, Bonjour avanza hacia las revoluciones más contemporáneas contra el enfoque clásico. Un libro sumamente versátil para adentrase, desde una perspectiva introductoria, en el estudio de la epistemología desde una filosofía receptiva.

Palabras clave: Bonjour, fundacionalismo, epistemología cartesiana, internalismo.

\section{Abstract:}

This text reflects Bonjour's conviction that classic problems are a point of departure for any discussions regarding theories of knowledge, which is where her interest in Descartes emerges from and upon which her attention is focused. After acknowledging the foundations for understanding epistemology, Bonjour advances toward more contemporary revolutions against the classic approach. This extremely versatile book allows the reader an introductory perspective from which to step into the study of epistemology using a receptive philosophy.

Key words: Bonjour, foundationalism, Cartesian epistemology, internalism. 
- sta traducción del libro de Laurence Bonjour corresponde a la segunda edición en inglés; su difusión es relevante no solo por el elevado costo del libro importado de Estados Unidos y lo difícil de adquirir para nuestros magros bolsillos, sino que también en esta edición en español, la primera en este idioma, se incluyen preguntas para pensar y discutir la epistemología, además de un glosario en cada uno de los doce capítulos que componen las dos partes en las que se divide esta obra. Este extra es agradable, importante e interesante ya que nos permite la realización de ejercicios en las aulas o bien, resolverlos solo como lectores interesados en la filosofía.

En la primera parte del libro, Bonjour - y su aporte a los problemas epistemológicos - inicia con el rescate de una propuesta cartesiana, centrada en cuatro principios fundamentales para comprender los supuestos y compromisos que establecerán la agenda y las líneas de investigación en y desde el conocimiento. Los cuatro principios establecen que:

1. El conocimiento supone una creencia aceptada por quien posea alguna razón que garantice su verdad,

2. El conocimiento tiene una base racional o a priori asentada en la autoevidencia,

3. El conocimiento con base empírica proviene de la experiencia inmediata,

4. La inferencia del mundo material y externo proviene del conocimiento racional y empírico (Bonjour, 2019:52-53).

El primero, desarrollado en el capítulo tres, manifiesta la noción misma del conocimiento con base en la definición cartesiana de creencia verdadera justificada. Estos elementos son extraídos por Bonjour para definir y exponer distintas posiciones a partir de su problematización y justificación.

El problema de la inducción es analizado en el capítulo cuatro, los problemas de otras mentes, del testimonio y de la memoria se originan a partir del cuarto principio.

En el capítulo cinco se deriva el problema de la justificación y el conocimiento a priori a partir del segundo principio, el cual integra de forma central la propuesta fundacionalista de Bonjour.

Con la intención de constituir una base para el conocimiento, en el capítulo seis el apriorismo enfatiza el problema de la experiencia inmediata. Derivado del tercer principio, que complementa el fundacionalismo de Descartes (2003), 
Bonjour desarrolla la justificación del conocimiento del mundo externo y esboza, de manera puntual, las distintas corrientes epistemológicas alternativas que han dado cuenta de la empresa fundacionalista cartesiana (Chirinos, 2013:131).

La segunda parte del libro nos muestra diversas corrientes contemporáneas de la filosofía, las cuales pretenden reconocer los problemas trazados en la primera parte (como la propuesta fundacionalista-internalista, defendida por Bonjour al inicio). En esta revisión de la epistemología, en los albores del siglo XXI, la Filosofía se define como la investigación del entendimiento reflexivo; es decir, ver cómo las cosas, en el sentido más extenso del término, se encajan mutuamente en la forma más amplia de la expresión. El filósofo está particularmente preocupado por temas o áreas generales: la naturaleza y composición de los seres humanos, los ingredientes básicos y la estructura de la realidad, la naturaleza y la base del valor.

La mayoría de los temas investigados por el filósofo también se pueden estudiar desde otros puntos de vista, en especial desde la perspectiva de la ciencia empírica. Pero si bien el filósofo puede hacer uso de los resultados de otras investigaciones, su enfoque es diferente: más general, más abstracto y dirigido de manera distintiva a los problemas intelectuales que surgen en el esfuerzo por comprender lugares donde nuestro pensamiento parece atado en nudos o enredos difíciles de desenredar, difíciles de entender. Es la presencia de problemas de este tipo lo que hace que un tema sea de especial interés para los filósofos. Resulta que ese conocimiento es un área temática en la cual los problemas son especialmente difíciles, omnipresentes y preocupantes en sus implicaciones.

En segundo término, para Bonjour, el modelo holístico de la teoría coherentista de la justificación es precisamente lo que se necesita para resolver el problema del retroceso infinito de las razones. Esto es así porque, según la concepción coherente, la justificación no se transfiere de una creencia que ya está justificada a otra que está esperando recibir justificación, a través de relaciones inferenciales (de prioridad epistémica), sino que es epistémicamente propiedad simultánea de todo el sistema de creencias, es decir, la propiedad de la coherencia del sistema en su conjunto. Solo si uno tuviera la antigua visión atomista, no holística, de la justificación empírica, que el coherentismo rechaza de forma explícita y resuelta, seguiría lo que muchos epistemólogos dan por sentado sin mucho argumento adicional a saber: que la justificación coherente es viciosamente circular.

Contrario a ello, Bonjour retoma su teoría coherentista de la justificación a partir de las siguientes premisas: a) la coherencia lógica no necesita ser absoluta- 
mente necesaria en la condición de la coherencia de un sistema; b) un sistema de creencias bien puede tener un alto grado de coherencia a pesar de la presencia de incoherencia local dentro de él; c) la coherencia de un sistema de creencias es proporcional al grado de su consistencia probabilística; d) la coherencia de un sistema de creencias aumenta, si esas creencias están relacionadas de manera inferencial, es decir, son parte de un proceso por el cual se derivan conclusiones a partir de premisas, y este aumento es directamente proporcional al número y la fuerza de las relaciones inferenciales en cuestión.

En tercer término, podemos señalar que la teoría coherentista de la justificación de un sistema de creencias disminuye si existen dentro de él varios subsistemas de creencias que están relativamente desconectados (es decir, su relación no es inferencial). Solo las relaciones inferenciales pueden proporcionar apoyo epistémico mutuo entre las creencias. Dos o más creencias están relacionadas inferencialmente si una puede servir como premisa de un argumento para la justificación de otra. Sin embargo, es fundamental entender que aunque una creencia esté bajo esta relación, eso no es suficiente para obtener la coherencia ideal, ya que no excluye la posibilidad de que dos o más sistemas conceptuales puedan ser coherentemente considerados de forma aislada, es decir, sin ser ellos mismos inferencialmente conectados. De forma intuitiva, parece que un sistema idealmente coherente es aquel en el que sus subsistemas, con relativa independencia, son conectados inferencialmente por leyes y principios explicativos de nivel superior.

En cuarto término, la teoría coherentista de la justificación de un sistema de creencias se reduce en un grado que es directamente proporcional a la presencia de anomalías inexplicables dentro de él. Esto significa que para una definición adecuada del concepto de coherencia debemos considerar la existencia de relaciones explicativas entre creencias (más allá de sus meras conexiones inferenciales). Bonjour intenta proporcionar una respuesta plausible a una de las preguntas más apremiantes que toda teoría de coherencia tiene que enfrentar tarde o temprano, es decir, dejar espacio para un concepto plausible de observación dentro de los límites de una teoría de coherencia. ¿Cómo puede funcionar la observación como un control epistémico independiente de otras creencias empíricas no observacionales del sistema, en el contexto de una teoría de coherencia generalizada de creencias empíricas?

En ausencia de esta función epistémica crucial de observación, parece que no hay nada en una teoría coherentista de la justificación que requiera que el contenido de las creencias empíricas dentro del sistema sea en última instancia 
provisto por el mundo externo, es decir, un mundo que exista y tenga ciertas características estructurales y cualitativas, propiedades más allá de su representación desde nuestro sistema de creencias.

La respuesta de Bonjour a este problema es que es posible identificar una subclase de creencias, es decir, las creencias cognitivas espontáneas (creencias de origen no inferencial) y deducir que algunos tipos de esas creencias cognitivas espontáneas, por ejemplo, los introspectivos u observacionales, en contraste con las creencias cognitivamente espontáneas, son el resultado de una ilusión, corazonadas o dogmas irracionales infundados. Son, desde el punto de vista del sistema de creencias del que forman parte, altamente confiables, por lo tanto, probablemente ciertos.

En quinto y último término, la creencia en la fiabilidad de los grupos de creencias cognitivamente espontáneos se basa en el hecho de que los miembros individuales del grupo están de acuerdo entre sí, así como con los miembros de otros grupos de creencias espontáneas. Este acuerdo consiste en la ausencia de anomalías entre ellos, en su unión con otros principios teóricos que contribuyen a la formulación de una imagen coherente cada vez mayor de una realidad independiente y objetiva y, de manera crucial, en la existencia de una hipótesis que explica su fiabilidad. Las creencias cognitivamente espontáneas que cumplen con los requisitos anteriores pueden considerarse, con justificación, como observacionales, mientras que las creencias no observacionales del sistema solo pueden justificarse si son coherentes con esas creencias observacionales.

Sin embargo, el propio Bonjour observa que, en esta etapa de su argumento, su teoría coherentista de la justificación solo permite la posibilidad de una aportación independiente externa al proceso interno de justificación coherente de nuestras creencias empíricas, no garantiza que este sea realmente el caso. Esto se debe a que es posible que un sistema de creencias que implique que ciertos tipos reconocibles de creencias cognitivamente espontáneas sean verdaderas, sin embargo, puede dejar de implicar que el contenido de esas creencias está correlacionado de manera confiable con causas mundanas externas.

Creemos, al igual que Bonjour, que una teoría coherentista de la justificación que pretenda ser una guía en esencia (y no solo accidentalmente) confiable de la verdad empírica debe requerir que las creencias individuales del sistema puedan ser candidatas para la justificación empírica solo si el sistema incluye leyes que atribuyan un alto grado de fiabilidad a una amplia variedad de creencias cognitivamente espontáneas. 
Por lo anterior, este libro, en su traducción al español, cumple de manera fiel un objetivo fundamental, una exposición de los enfoques y teorías filosóficas de forma clara y profunda, con sus referentes críticos y problemas internos, lo cual estimula al lector a debatir cada enfoque a través de preguntas al final de cada capítulo. Desde esta perspectiva, la Filosofía es substancialmente dialéctica, fundada en tesis y antítesis, que nos llevan a repasar y repensar estrategias pedagógicas en las aulas de clase: la idea de aprender una Filosofía crítica y reflexiva a partir de problemas a ras de suelo y no desde el desapego que los autores o las teorías pueden producir en el joven lector.

\section{Bibliografía citada}

Bonjour, Laurence. (2010). Epistemology. Classic Problems and Contemporary Responses. United Kingdom: Rowman \& Littlefield Publishers, Inc.

Chirinos Bossio, Ricardo. (2013). Antifundacionalismo y holismo cognoscitivo en

Richard Rorty. Opción, 29(71), mayo-agosto, pp. 125-148.

Descartes, René. (2003). Reglas para la dirección del espíritu. Madrid: Alianza Editorial.

\section{Cómo citar esta reseña:}

